AperTO - Archivio Istituzionale Open Access dell'Università di Torino

\title{
Automatic method for tumor segmentation from 3-points dynamic PET acquisitions
}

\section{This is the author's manuscript}

Original Citation:

\section{Availability:}

This version is available http://hdl.handle.net/2318/154166

since 2016-06-16T00:46:00Z

Publisher:

IEEE

Published version:

DOI:10.1109/ICIP.2014.7025188

Terms of use:

Open Access

Anyone can freely access the full text of works made available as "Open Access". Works made available under a Creative Commons license can be used according to the terms and conditions of said license. Use of all other works requires consent of the right holder (author or publisher) if not exempted from copyright protection by the applicable law. 
This is the author's final version of the contribution published as:

Francesco Verdoja; Marco Grangetto; Christian Bracco; Teresio Varetto; Manuela Racca; Michele Stasi. Automatic method for tumor segmentation from 3-points dynamic PET acquisitions, in: IEEE International Conference on Image Processing, IEEE, 2014, 9781479957507, pp: 937-941.

The publisher's version is available at:

http://xplorestaging.ieee.org/ielx7/6992914/7024995/07025188.pdf?arnumber=7025188

When citing, please refer to the published version.

Link to this full text:

http://hdl.handle.net/2318/154166 


\section{AUTOMATIC METHOD FOR TUMOR SEGMENTATION FROM 3-POINTS DYNAMIC PET ACQUISITIONS}

\author{
Francesco Verdoja, Marco Grangetto \\ Università degli Studi di Torino \\ Computer Science department \\ Corso Svizzera 185, Torino, Italy
}

\author{
Christian Bracco $^{\dagger}$, Teresio Varetto $^{\ddagger}$, Manuela Racca $^{\ddagger}$, Michele Stasi $^{\dagger}$ \\ Istituto per la Ricerca e la Cura del Cancro (IRCC) \\ $\dagger$ Medical Physics department, $\ddagger$ Nuclear Medicine department \\ SP $142 \mathrm{~km}$ 3.95, Candiolo (TO), Italy
}

(1)

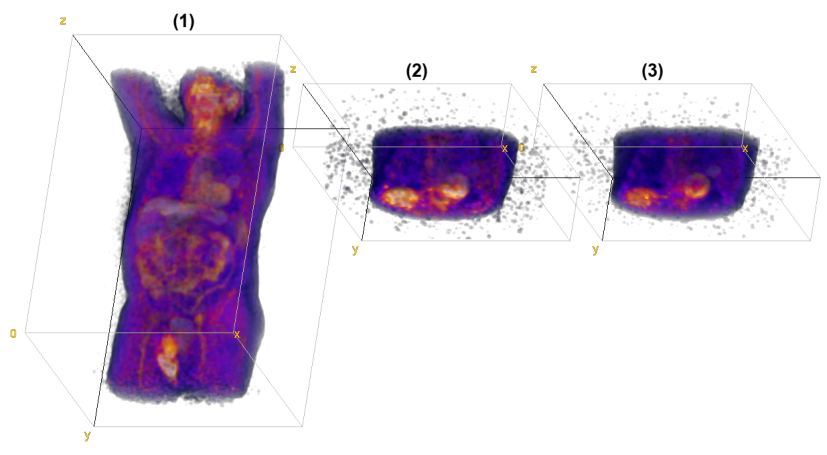

In this paper a novel technique to segment tumor voxels in dynamic positron emission tomography (PET) scans is proposed. An innovative anomaly detection tool tailored for 3-points dynamic PET scans is designed. The algorithm allows the identification of tumoral cells in dynamic FDG-PET scans thanks to their peculiar anaerobic metabolism experienced over time. The proposed tool is preliminarily tested on a small dataset showing promising performance as compared to the state of the art in terms of both accuracy and classification errors.

Index Terms - Medical diagnostic imaging, image segmentation, anomaly detection, positron emission tomography, tumors

\section{INTRODUCTION}

In oncology, the ability to properly contour tumors in PET/CT images is clinically important as treatment plans depend on information on the tumor volume. The tumor cells should be identified as precisely as possible since underestimation or overestimation of their volume can lead to treatments that can be either ineffective or dangerous [1].

Manual contouring has been proven to be subjective and inaccurate [2] and therefore the need for an automatic method for tumor region segmentation in PET has arisen. However, PET segmentation remains an open problem mainly because of the limited image resolution and presence of acquisition noise [3].

For these reasons, many techniques for automatic or semiautomatic image segmentation have been proposed. However validation of accuracy and precision of these algorithms is still unresolved, due also to the lack of standard approaches and guidelines by nuclear medicine and radiation oncology professional societies [3].

In this paper, a novel approach based on anomaly detection technique is proposed. Using 3 PET images acquired at different time instants, we aim at detecting tumoral voxels recognizing their anomalous bahavior over time. The contributions of the paper are the design of a novel anomaly detection tool tailored for dynamic PET scans and a preliminary experimentation on a set of PET scans acquired at the Institute for Cancer Research and Treatment of Candiolo (IRCC). Our findings show that the proposed approach is very promising and competitive with respect to the state of the art even if the number of tested subjects was limited by the fact that 3-points dynamic PET scan represents a frontier technology that is not part of any standard clinical protocol at the moment.
Fig. 1. The three 3D PET images of one of the sample patients; (1) is the early full body scan $(E S, 144 \times 144 \times 213 \mathrm{px}),(2)$ and (3) are the two 5 minutes reconstructions of the delayed scan $(D S 1$ and $D S 2,144 \times 144 \times 45 \mathrm{px})$, where only the area around the tumor is acquired. These greyscaled images are here displayed using a Fire lookup table.

\section{BACKGROUND}

Positron emission tomography using fluorodeoxyglucose as tracker (known as FDG-PET) is mainly based on tissue metabolic activity by virtue of the regional glucose uptake.

Due to multiple mutations in the DNA occurred during cell replication, cancer cells lack the ability to stop their duplication after a certain point, raising cell density in tumor region and leading to insufficient blood supply. The resulting deficiency of oxygen (hypoxia) force the cells to rely mostly on the anaerobic metabolism, i.e. glycolysis [4]. As a consequence, glycolysis can be used as an excellent marker in the detection of cancer cells; FDG-PET - in which the concentrations of the tracer indicate a glucose uptake in the imaged area - turns to be a good tool in detecting tumoral masses, lymph nodes and cancer metastasis all at once [5].

In images obtained by PET acquisitions the intensity of a voxel represents local concentration of the tracer. The most commonly used unit is called SUV (Standardized Uptake Value) which is defined as the ratio of the tissue radioactivity concentration over the injected activity divided by the body weight [6]. It aims to be a quantitative measure of tracer uptake that can be compared between patients, but it is also criticized for its misuse [7].

There are two classes of PET scans: static and dynamic. In static mode, accounting for the majority of PET scans used nowadays [8], the tracer uptake is integrated over a single acquisition producing a single value per imaged volume (voxel). In the case of a dynamic 
(a)

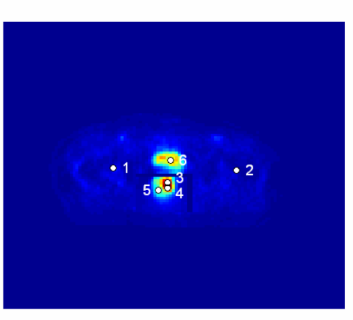

(b) $5^{\times}$

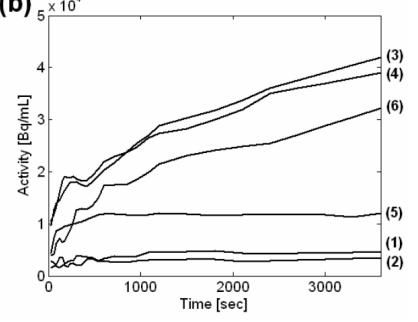

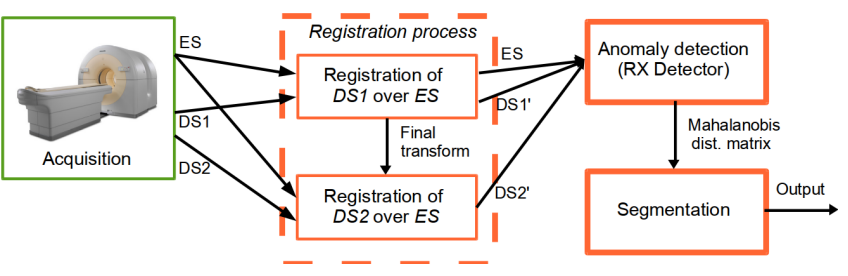

Fig. 3. Flowchart of the proposed technique

Fig. 2. In (a) six points are chosen on a PET slice: two points within the normal tissue ( 1 and 2 ), two points within the tumor ( 3 and 4 ), one point at the boundary of the tumor (5) and one point within the bladder (6). In $(b)$ the TACs of the selected points resulting from a dynamic PET scan are shown. Image courtesy of [8].

scan, tracer activity is measured in different time windows, resulting in a time activity curve (TAC) for each voxel [9]. The shape of the TACs, usually found by sample interpolation, conveys tissue specific biochemical properties over time and carries precious information on the amount and rate of tracer flux and accumulation [10].

In static PET, the most common techniques that have been proposed for automatic or semiautomatic tumor segmentation are thresholding algorithms, where a threshold value $(T)$ on the SUV is selected to separate the tumor from the background [11]. Other types of techniques that have been proposed for static PET are variational approaches based on deformable active contours [12], stochastic models mainly based on Expectation-Maximization (EM) algorithm [13] and learning methods with and without supervision.

Segmentation in dynamic PET involves analysis of TACs instead of single voxel values; in this way the temporal information is used to improve quality of delineation [14]. Clustering techniques have been proposed in literature $[2,15]$. In this group of techniques, $F C M-S W$ is an approach based on the Fuzzy $c$-Means algorithm that is reported to perform quite well [16]. Stochastic approaches have been followed, as well: a mixture model has been proposed that expressed a voxel-level TAC as a weighted combination of scaled sub-TACs [17]. However, these methods did not consider spatial relationship among voxels. Some algorithms including spatial information have been proposed [18, 19], but most of these methods were designed for brain images where regions have similar dimensions and are rather inefficient in the case of whole body images where sizes are quite different [14]. Recently, a local means analysis method was proposed; it uses a hierarchical linkage algorithm with a seed growing method [20].

\section{THE PROPOSED TECHNIQUE}

In this paper a novel approach for automatic tumor segmentation in dynamic PET images is proposed. The algorithm works on two PET scans where the second acquisition (10 minutes long) is taken after about an hour from the first one. Every acquisition can be reconstructed in a variable number of images, each one collecting events occurred in a given time window. For this study the first scan has been reconstructed into a single full body image (called early scan, $E S$ ) and the second one is used to construct two images (delayed scans, $D S 1$ and $D S 2$ ), collecting respectively events occurred in the first 5 minutes and in the last 5 minutes of the second acquisition and imaging only the area in which the tumor is expected to be. Therefore our proposal is based on the automatic analysis of 3 PET images (Figure 1).

The proposed algorithm exploits the assumption that in cancer cells the glucose uptake over time is very different from the one in normal tissues [4]; as a consequence, we propose to use a statistical anomaly detection mechanism able to detect voxel with peculiar temporal behavior, i.e. anomalous TAC. An example of this phenomenon can be seen in Figure 2.

Although, to the best of our knowledge, methodologies of this kind have never been proposed for PET scans, algorithms based on anomaly detection have already been used in other medical domains, e.g. on CT images [21] and for segmentation in endoscopic video sequences [22].

The block diagram showing the main steps of the proposed algorithm is shown in Figure 3. Since PET scans acquired at different time instants are going to be used, the first processing stage is represented by image registration. In fact, the patient has left the scanner bed between the scans, and then he/she has obviously slightly changed his/her position between the first and the second scan. Registration of $D S 1$ and $D S 2$ with respect to $E S$ is therefore required.

\subsection{Registration}

The registration process consists in the application of a transformation to align a moving image over a fixed one (in this study $E S$ ). The transformation parameters are initialized and then refined by an optimizer according to a metric. The final transformation is then applied to the moving image using interpolation.

Since no deformation is expected, and the image has been just translated and rotated, affine transformation has been used. It is the most common choice in instances of rigid-body movement [23]. Then, linear interpolation can be employed for registration, under the assumtion that intensities vary linearly between grid positions as discussed in [23]. Finally, normalized cross-correlation has been employed as registration metric and it is optimized by a gradient descent approach; this combination is suggested to work well on full body monomodal PET-PET registrations [24].

The computational cost for the registration of $D S 2$ can be reduced noticing that both $D S 1$ and $D S 2$ are reconstructed from the same scan and therefore they share almost the same acquisition conditions. As a consequence, the transformations leading to registration of $D S 1$ and $D S 2$ are expected to be very similar. For this reason, to limit computation, we first register $D S 1$; then, the final transformation obtained on $D S 1$ is provided as an initial estimate for $D S 2$ registration. This solution allowed us to cut by one half the number of iterations required to register $D S 2$.

Let us refer to the two registered images as $D S 1^{\prime}$ and $D S 2^{\prime}$; their voxels can be considered as co-located with those of $E S$ and represent the input of the core part of the proposed tool, i.e. the anomaly detection stage. 


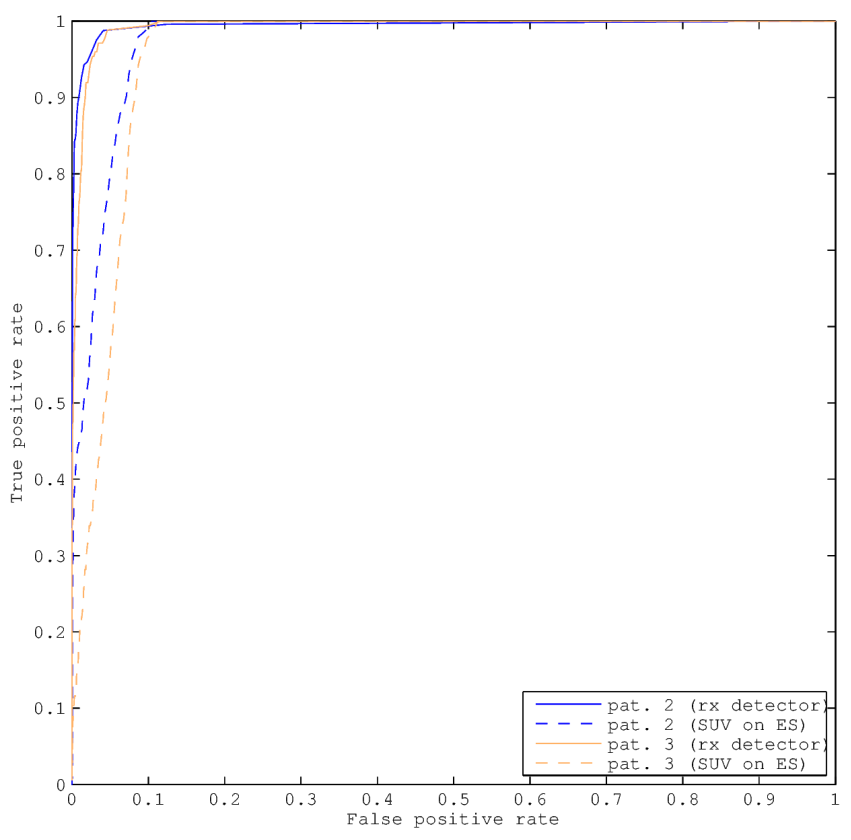

Fig. 4. ROC curves of proposed methods and simple SUV thresholding over two sample patients.

\subsection{Anomaly detection}

The goal of our algorithm is to identify those voxels exhibiting an anomalous tracer uptake over time. To this end, we employ the RX Detector [25] as follows.

Let us introduce the row vector $x_{i}=\left(x_{i, E S}, x_{i, D S 1^{\prime}}, x_{i, D S 2^{\prime}}\right)$ representing the $3 \mathrm{SUV}$ values of the $i$-th voxel of $E S, D S 1^{\prime}$ and $D S 2^{\prime}$ respectively. The typical behavior of the normal voxels can be captured by the covariance matrix that can be estimated as:

$$
\widehat{C}=\frac{1}{N} \sum_{i=1}^{N}\left(x_{i}-M\right)\left(x_{i}-M\right)^{T}
$$

where $N$ is the total number of voxels in the image volume and $M=$ $\left(\mu_{E S}, \mu_{D S 1^{\prime}}, \mu_{D S 2^{\prime}}\right)$ is the average value of the 3 components.

The covariance matrix is computed assuming that vectors $x_{i}$ are observations of the same random process. Under the legitimate assumtion that normal voxels represent the majority of the acquired data, it can be concluded that the covariance matrix estimated using all voxels is representative of the healty tissues [26].

According to the RX Detector the generalized likelihood ratio test for identifying anomalous voxel with respect to the covariance model $\widehat{C}$ can be expressed as:

$$
\delta_{R X}\left(x_{i}\right)=\left(x_{i}-M\right)^{T} \widehat{C}^{-1}\left(x_{i}-M\right)>\eta
$$

where $\eta$ is a proper decision threshold. It is worth pointing out that $\delta_{R X}$ is also known as Mahalanobis distance [27].

The 3D image associating the value of $\delta_{R X}$ to each voxel can also be interpreted as a Mahalanobis distance matrix that takes higher values if the TAC exhibits an anomalous profile.

In this preliminary work we propose to detect the tumor voxels setting the decision threshold $\eta$ adaptively as a function of the $\delta_{R X}$ dynamic range. Therefore we set $\eta=P \cdot \max \left(\delta_{R X}\right)$ with $P \leq 1$. We leave the design of optimal decision mechanisms, taking into
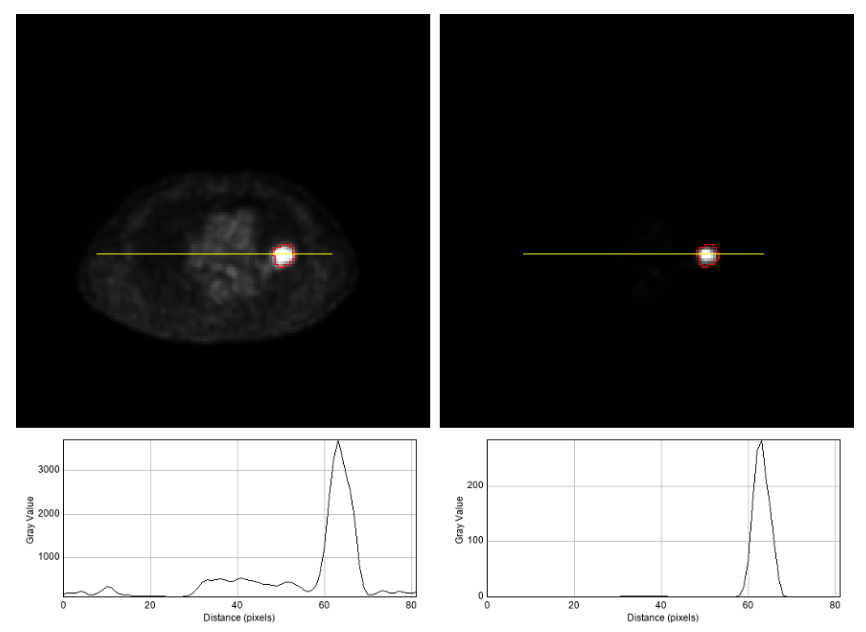

Fig. 5. The same slice in $E S$ (left) and $\delta_{R X}$ (right) with manual contour of tumor area superimposed (red line). Under each image a $2 \mathrm{D}$ plot of the intensities over the yellow line is presented.

account also spatial consistency of the selected tumor volume, for future research.

\section{EXPERIMENTAL VALIDATION}

As already mentioned, the novelty of the proposed approach is two-fold, namely the usage on 3-points dynamic PET scans and the exploitation of the RX Detector for tumor contouring. Since nowadays dynamic PET scans are not used on a large scale for clinical treatment, our findings are limited to a small dataset, comprising 6 subjects, that has been made available at the IRCC for research purpose. A Philips Gemini TF PET/CT has been used for acquiring both CT and FDG-PET scans for all the patients. To this end we acknowledge the precious cooperation of nuclear medicine physicians that have manually segmented the PET images, setting up the ground truth for computation of the accuracy yielded by the proposed automatic tool.

In Figure 4 the Receiver Operating Characteristic (ROC), i.e. the true positive rate (TPR) versus the false positive rate (FPR), obtained classifying voxels according to (2) varying the value of $\eta$ is shown. The ROC curves computed this way are compared with those obtained with simple thresholding of the voxel SUV values. It can be noted that using anomaly detection the detection accuracy significantly improves with respect to the original SUV domain segmentation.

In Figure 5 the original SUV image (left) and $\delta_{R X}$ values (right) of a PET section are shown. It can be clearly observed that the $\delta_{R X}$ domain is very effective in identifying the target tumoral region.

The proposed segmentation results have been evaluated using objective metrics as well, namely in terms of the tumoral volume estimates and the Spatial Overlap Index (SOI) defined in [28] as

$$
S O I=\frac{2(A \cap B)}{A+B}
$$

where, when $A$ and $B$ are two binary masks (i.e. manual ROI and segmented output of RX Detector), the intersection operator is used to indicate the number of voxels having value 1 in both masks and the sum operator the total number of voxels having value 1 in the two masks. 


\begin{tabular}{cccccc}
\hline & \multicolumn{2}{c}{ SOI } & \multicolumn{3}{c}{ Volumes (ml) } \\
n. & RX & Thresh. & RX & Thresh. & ROI \\
\hline 1 & 0.569 & 0.659 & 7.808 & 9.856 & 19.648 \\
2 & 0.564 & 0.416 & 12.032 & 13.568 & 15.680 \\
3 & 0.377 & 0.029 & 24.512 & 248.128 & 11.136 \\
4 & 0.674 & 0.000 & 11.072 & 3.136 & 6.592 \\
5 & 0.628 & 0.000 & 11.072 & 3.136 & 7.872 \\
6 & 0.433 & 0.514 & 2.816 & 6.976 & 7.232 \\
\hline \hline Mean & 0.541 & 0.270 & 11.552 & 47.467 & 11.360 \\
Std Dev & 0.114 & 0.295 & 7.197 & 98.386 & 5.279 \\
\hline
\end{tabular}

Table 1. SOI and tumoral volume estimate of proposed RX Detector $(P=0.27)$ and SUV thresholding $(P=0.38)$.

The results are summarized in Table 1, where SOI and volume estimate obtained by the proposed technique and SUV thresholding are compared. The experiments have been worked out setting different thresholds values. Table 1 shows the results corresponding to the thresholds yielding the best average SOI, i.e. $P=0.27$ and $P=0.385$ for $\delta_{R X}$ and SUV segmentation respectively. It can be observed that the proposed method offers the best average results both in terms of SOI and volume estimate. In particular, it is worth pointing out that SUV thresholding completely fails on patients 3 , 4 and 5 whereas the proposed tool yields significant results on the whole dataset.

Our results turns to be competitive with those reported in [3], where a set of segmentation techniques are compared in terms of SOI using a dataset of 7 patients suffering from pharyngolaryngeal squamous cell carcinoma. In particular, in [3] it is found that one of the best performing method, termed $F C M-S W$, yields an average SOI of 0.54. FCM-SW employs a more complex segmentation approach as opposed to our simple 3-points RX Detector: it is based on fuzzy $c$-Means algorithm coupled with anisotropic diffusion filter to incorporate spatial information and wavelet transform. Even if the results are computed on different datasets it is noticeable that the proposed simpler algorithm achieve comparable SOI performance.

\section{CONCLUSIONS}

In this paper a novel technique for the automatic detection of tumoral volumes in 3-points TACs has been designed and preliminarily investigated using a dataset comprising 6 patients. The proposed approach leverages on the well known RX Detector, applied for the first time to this domain, to look for anomalies in 3-points TAC. The basic assumption is that tumor and background regions have different uptake curves over time that can be discriminated using 3-points dynamic PET acquisitions.

Our experimentation in the field confirms that RX Detector effectively improves the quality of the segmentation by significantly enhancing contrast between tumor region and background. In this preliminary study segmentation has been performed with a naïve approach based on simple thresholding; nonetheless the achieved SOI and volume estimates are in line with the results reported in the literature based on more complex segmentation mechanisms. Therefore, we believe that our study paves the way to further improvements using more accurate segmentation strategies founded on RX Detector.

Although results seem encouraging, future validation of the method should be performed to be able to confirm all the hypothesis contained in this work. This validation should evaluate the algorithm performance over a larger data set, considering a wider range of tumor volumes.

\section{REFERENCES}

[1] Carlos A. Perez and Luther W. Brady, Principles and practice of radiation oncology, Lippincott Williams \& Wilkins, Philadelphia, PA, 5th ed. edition, 2008.

[2] Koon-Pong Wong, Dagan Feng, Steven R. Meikle, and Michael J. Fulham, "Segmentation of dynamic PET images using cluster analysis," IEEE Transactions on Nuclear Science, vol. 49, no. 1, pp. 200-207, Feb. 2002.

[3] Habib Zaidi, Mehrsima Abdoli, Carolina Llina Fuentes, and Issam M. El Naqa, "Comparative methods for PET image segmentation in pharyngolaryngeal squamous cell carcinoma," European Journal of Nuclear Medicine and Molecular Imaging, vol. 39, no. 5, pp. 881-891, Jan. 2012.

[4] Bruce Alberts, Alexander Johnson, Julian Lewis, Martin Raff, Keith Roberts, and Peter Walter, Molecular biology of the cell, Garland Science, New York, NY, 5th ed. edition, Dec. 2007.

[5] Ken Garber, "Energy boost: The warburg effect returns in a new theory of cancer," JNCI Journal of the National Cancer Institute, vol. 96, no. 24, pp. 1805-1806, Dec. 2004.

[6] Giovanni Lucignani, Giovanni Paganelli, and Emilio Bombardieri, "The use of standardized uptake values for assessing FDG uptake with PET in oncology: a clinical perspective," $\mathrm{Nu}$ clear Medicine Communications, vol. 25, no. 7, pp. 651-656, July 2004, PMID: 15208491.

[7] John W. Keyes, "SUV: standard uptake or silly useless value?," The Journal of Nuclear Medicine, vol. 36, no. 10, pp. 18361839, Oct. 1995, PMID: 7562051.

[8] Marco H.M. Janssen, Hugo J.W.L. Aerts, Michel C. llers, Geert Bosmans, John A. Lee, Jeroen Buijsen, Dirk De Ruysscher, Philippe Lambin, Guido Lammering, and Andre L.A.J. Dekker, "Tumor delineation based on TimeActivity curve differences assessed with dynamic fluorodeoxyglucose positron emission TomographyComputed tomography in rectal cancer patients," International Journal of Radiation Oncology*Biology*Physics, vol. 73, no. 2, pp. 456-465, Feb. 2009.

[9] Catherine J. Kelly and Michael Brady, "A model to simulate tumour oxygenation and dynamic [18F]-Fmiso PET data," Physics in medicine and biology, vol. 51, no. 22, pp. 58595873, Nov. 2006, PMID: 17068369.

[10] Daniela Thorwarth, Susanne M. Eschmann, Frank Paulsen, and Markus Alber, "A kinetic model for dynamic [18F]-Fmiso PET data to analyse tumour hypoxia," Physics in medicine and biology, vol. 50, no. 10, pp. 2209-2224, May 2005, PMID: 15876662.

[11] Yusuf E. Erdi, Osama Mawlawi, Steven M. Larson, Massimo Imbriaco, Henry Yeung, Ronald D. Finn, and John L. Humm, "Segmentation of lung lesion volume by adaptive positron emission tomography image thresholding," Cancer, vol. 80, no. 12 Suppl, pp. 2505-2509, Dec. 1997, PMID: 9406703.

[12] Stanley Osher and James A. Sethian, "Fronts propagating with curvature dependent speed: Algorithms based on hamiltonjacobi formulations," Journal of Computational Physics, vol. 79, no. 1, pp. 1249, 1988.

[13] Michalis Aristophanous, Bill C. Penney, Mary K. Martel, and Charles A. Pelizzari, "A gaussian mixture model for definition of lung tumor volumes in positron emission tomography," Medical physics, vol. 34, no. 11, pp. 4223-4235, Nov. 2007, PMID: 18072487. 
[14] Jinxiu Cheng-Liao and Jinyi Qi, "Segmentation of mouse dynamic PET images using a multiphase level set method," Physics in Medicine and Biology, vol. 55, no. 21, pp. 65496569, Nov. 2010.

[15] Jovan G. Brankov, Nikolas P. Galatsanos, Yongyi Yang, and Miles N. Wernick, "Segmentation of dynamic PET or fMRI images based on a similarity metric," IEEE Transactions on Nuclear Science, vol. 50, no. 5, pp. 1410-1414, Oct. 2003.

[16] Saoussen Belhassen and Habib Zaidi, "A novel fuzzy c-means algorithm for unsupervised heterogeneous tumor quantification in PET," Medical Physics, vol. 37, no. 3, pp. 1309-1324, Mar. 2010.

[17] Finbarr O'Sullivan, "Locally constrained mixture representation of dynamic imaging data from PET and MR studies," Biostatistics, vol. 7, no. 2, pp. 318-338, 2006.

[18] Jinman Kim, Weidong Cai, Dagan Feng, and Stefan Eberl, "Segmentation of VOI from multidimensional dynamic PET images by integrating spatial and temporal features," IEEE Transactions on Information Technology in Biomedicine, vol. 10, no. 4, pp. 637-646, Oct. 2006.

[19] Brian J. Parker and Dagan Feng, "Graph-based mumford-shah segmentation of dynamic PET with application to input function estimation," IEEE Transactions on Nuclear Science, vol. 52, no. 1, pp. 79-89, Feb. 2005.

[20] Renaud Maroy, Raphal Boisgard, Claude Comtat, Vincent Frouin, Pascal Cathier, Edouard Duchesnay, Frdric Doll, Peter E Nielsen, Rgine Trbossen, and Bertrand Tavitian, "Segmentation of rodent whole-body dynamic PET images: an unsupervised method based on voxel dynamics," IEEE transactions on medical imaging, vol. 27, no. 3, pp. 342-354, Mar. 2008, PMID: 18334430.

[21] Aminmohammad Roozgard, Samuel Cheng, and Hong Liu, "Malignant nodule detection on lung CT scan images with kernel RX-algorithm," in International Conference on Biomedical and Health Informatics (BHI), Hong Kong and Shenzhen, China, Jan. 2012, pp. 499-502, IEEE.

[22] Barbara Penna, Tammam Tillo, Marco Grangetto, Enrico Magli, and Gabriella Olmo, "A technique for blood detection in wireless capsule endoscopy images," in 17th European Signal Processing Conference (EUSIPCO), Glasgow, Scotland, Aug. 2009, pp. 1864-1868, EURASIP.

[23] J. B. Antoine Maintz and Max A. Viergever, "A survey of medical image registration," Medical Image Analysis, vol. 2, no. 1, pp. 1-36, Mar. 1998.

[24] Jesper L. R. Andersson, Anders Sundin, and Sven Valind, "A method for coregistration of PET and MR brain images," The Journal of Nuclear Medicine, vol. 36, no. 7, pp. 1307-1315, July 1995, PMID: 7790961.

[25] Irving S. Reed and Xiaoli Yu, "Adaptive multiple-band CFAR detection of an optical pattern with unknown spectral distribution," IEEE Transactions on Acoustics, Speech, and Signal Processing, vol. 38, no. 10, pp. 1760-1770, Oct. 1990.

[26] Chein-I Chang and Shao-Shan Chiang, "Anomaly detection and classification for hyperspectral imagery," IEEE Transactions on Geoscience and Remote Sensing, vol. 40, no. 6, pp. 1314-1325, June 2002.

[27] Prasanta C. Mahalanobis, "On the generalized distance in statistics," in National Institute of Sciences of India, Calcutta, India, 1936, vol. 2, pp. 49-55.
[28] Kelly H. Zou, Simon K. Warfield, Aditya Bharatha, Clare M. C. Tempany, Michael R. Kaus, Steven J. Haker, William M. Wells, Ferenc A. Jolesz, and Ron Kikinis, "Statistical validation of image segmentation quality based on a spatial overlap index1," Academic Radiology, vol. 11, no. 2, pp. 178-189, Feb. 2004. 